\title{
A PDE Method to Segment Image Linear Objects with Application to Lens Distortion Removal
}

\author{
Moumen T. El-Melegy and Nagi H. Al-Ashwal \\ Electrical Engineering Department, \\ Assiut University, Assiut 71516, Egypt \\ moumen@aun.edu.eg
}

Received 23 October 2006; accepted 3 October 2007

\begin{abstract}
In this paper, we propose a partial differential equation based method to segment image objects, which have a given parametric shape based on energy functional. The energy functional is composed of a term that detects object boundaries and a term that constrains the contour to find a shape compatible with the parametric shape. While the shape constraints guiding the PDE may be determined from object's shape statistical models, we demonstrate the proposed approach on the extraction of objects with explicit shape parameterization, such as linear image segments. Several experiments are reported on synthetic and real images to evaluate our approach. We also demonstrate the successful application of the proposed method to the problem of removing camera lens distortion, which can be significant in medium to wide-angle lenses.
\end{abstract}

Key Words: Variational Methods, Partial Differential Equations, Level Sets, Image Segmentation, Hough Transform, Fuzzy Memberships, Radial Distortion, Lens Distortion Calibration.

\section{Introduction}

Variational methods and partial differential equations (PDEs) are more and more being used to analyze, understand and exploit properties of images in order to design powerful application techniques, see for example $[15,16,17]$. Variational methods formulate an image processing or computer vision problem as an optimization problem depending on the unknown variables (which are functions) of the problem. When the optimization functional is differentiable, the calculus of variations provides a tool to find the extremum of the functional leading to a PDE whose steady state gives the solution of the imaging or vision problem. A very attractive property of these mathematical frameworks is to state well-posed problems to guarantee existence, uniqueness and regularity of solutions [16]. More recently, implicit level set based representations of a contour [9] have become a popular framework for image segmentation [10,11, 1].

The integration of shape priors into PDE based segmentation methods has been a focus of research in past years $[2,3,4,5,6,7,8,12,13,14]$. Almost all of these variational approaches address the segmentation of non-parametric shapes in images. They use training sets to introduce the shape prior to the problem formulation in such a way that only familiar structures of one given object can be recovered. They typically do not permit the segmentation of several instances of the given object. This may be attributed to the fact that a level set function is restricted to the separation of two regions. As soon as more than two regions are considered, the level set idea looses parts of its attractiveness. These level-set methods find their largest area

Correspondence to: <moumen@aun.edu.eg>

Recommended for acceptance by Dr. Sporring

ELCVIA ISSN: 1577-5097

Published by Computer Vision Center / Universitat Autonoma de Barcelona, Barcelona, Spain 
of application in the segmentation of medical images. After all, none can expect to find two instances of a human heart in a patient's scanned chest images!

On the other hand, extracting image parametric shapes and their parameters is an important problem in several computer vision applications. For example, extraction of a line is a crucial problem in calculating lens distortion and matching in stereo pairs [18]. As such, our research has addressed the application of variational methods and PDEs to the extraction of linear shapes from images. To the best of our knowledge, we are not aware of any efforts, other than ours, in that regard. Towards this end, we associate the parameters of the linear shape within the energy functional of an evolving level set. While existing approaches do not consider the extraction of more than one object instance in an image, the case where they would fail, our formulation allows the segmentation of multiple linear objects from an image.

The basic idea of this paper is inspired by a level set formulation of Chan-Vese [1]. We introduce line parameters into a level set formulation of a Chan-Vese like functional in a way that permits the simultaneous segmentation of several lines in an image. The parameters of the line are not specified beforehand, they rather evolve in an unsupervised manner in order to automatically select the image regions that are linear and the parameters of each line are calculated. In particular, we will show that this approach allows detecting image linear segments while ignoring other objects. This simple, easy-to-implement method provides noiserobust results because it relies on a region-based driving flow.

Moreover we apply the proposed PDE-based level set method to the calibration and removal of camera lens distortion, which can be significant in medium to wide-angle lenses. Applications that require 3-D modelling of large scenes typically use cameras with such wide fields of view [18]. In such instances, the camera distortion effect has to be removed by calibrating the camera's lens distortion and subsequently undistorting the input image. One key feature of our method is that it integrates the extraction of image features needed for calibration and the computation of distortion parameters within one energy functional, which is minimized during level set evolution. Thus our approach, unlike most other nonmetric calibration methods [21, 22, 23], avoids the propagation of errors in feature extraction onto the computation stage. This results in a more robust computation even at high noise levels.

The organization of this paper is as follows: In Section 2, we briefly review a level set formulation of the piecewise-constant Mumford-Shah functional, as proposed in [1]. In Section 3, we augment this variational framework by a parametric term that affects the evolution of the level set function globally for one object in the image. In Section 4, we extend this in order to handle more than one parametric object. In Section 5 we describe several experiments to evaluate the proposed method. We apply this method to lens distortion removal in Section 6. The conclusions are presented in Section 7.

\section{Region-Based Segmentation with Level Sets and PDEs}

In [1] Chan and Vese detailed a level set implementation of the Mumford-Shah functional, which is based on the use of the Heaviside function as an indicator function for the separate phases. The Chan-Vese method used a piecewise-constant, region-based formulation of the functional, which allows the contour to converge to the final segmentation over fairly large distances, while local edge and corner information is well preserved. It can detect cognitive contours (which are not defined by gradients), and contours in noisy images.

According to the level-set framework a contour, $C$, is embedded in a single level set function $\phi: \Omega \rightarrow \Re$ such that:

$$
\left\{\begin{array}{l}
C=\{(x, y) \in \Omega: \phi(x, y)=0\}, \\
\text { inside }(C)=\{(x, y) \in \Omega: \phi(x, y)>0\}, \\
\text { outside }(C)=\{(x, y) \in \Omega: \phi(x, y)<0\}
\end{array}\right.
$$

In the Mumford-shah model, a piecewise constant segmentation of an input image $f$ is given by [1]:

$$
\begin{aligned}
& E_{\text {seg }}\left(c_{1}, c_{2}, \phi\right)=\mu \int_{\Omega}\left|\nabla H_{\varepsilon}(\phi)\right| d x d y+v \int_{\Omega} H_{\varepsilon}(\phi) d x d y \\
& \quad+\lambda_{1} \int_{\Omega}\left|f-c_{1}\right|^{2} H_{\varepsilon}(\phi) d x d y+\lambda_{2} \int_{\Omega}\left|f-c_{2}\right|^{2}\left(1-H_{\varepsilon}(\phi)\right) d x d y,
\end{aligned}
$$


where $c_{1}$ and $c_{2}$ are the mean values of the image $f$ inside and outside the curve defined as the zero-level set of $\phi$, respectively, and $\mu, v, \lambda_{1}, \lambda_{2}$ are regularizing parameters to be estimated or chosen a priori. $H_{\varepsilon}$ is the regularized Heaviside function defined as [1]

$$
H_{\varepsilon}(s)=\frac{1}{2}\left(1+\frac{2}{\pi} \arctan \left(\frac{s}{\varepsilon}\right)\right) .
$$

so

$$
\delta_{\varepsilon}(s)=\frac{d H_{\varepsilon}}{d s}=\frac{1}{\pi} \frac{\varepsilon}{\varepsilon^{2}+s^{2}} .
$$

The regularized $H_{\varepsilon}$ and $\delta_{\varepsilon}$ having a discretization with a support larger than zero permit the detection of interior contours - for example if one wants to segment a ring-like structure, starting from an initial contour located outside the ring.

The Euler-Lagrange equation for this functional is implemented in [1] by the following gradient descent:

$$
\frac{\partial \phi}{\partial t}=\delta_{\varepsilon}(\phi)\left[\mu \operatorname{div}\left(\frac{\nabla \phi}{|\nabla \phi|}\right)-v-\lambda_{1}\left(f-c_{1}\right)^{2}+\lambda_{2}\left(f-c_{2}\right)^{2}\right],
$$

where the scalars $c_{1}$ and $c_{2}$ are updated with the level set evolution and given by:

$$
\begin{aligned}
& c_{1}=\frac{\int f(x, y) H_{\varepsilon}(\phi) d x d y}{\int H_{\varepsilon}(\phi) d x d y}, \\
& c_{2}=\frac{\int f(x, y)\left(1-H_{\varepsilon}(\phi)\right) d x d y}{\int\left(1-H_{\varepsilon}(\phi)\right) d x d y} .
\end{aligned}
$$

Figure 1 illustrates the main advantages of this level set method. Minimization of the functional (2) is done by alternating the two steps of iterating the gradient descent for the level set function $\phi$ as given by (5) and updating the mean gray values for the two phases, as given in equations $(6,7)$. Implicit representation allows the boundary to perform splitting and merging.

\section{PDE Method for Line Segmentation}

Our goal here is to extend the energy functional (2) in order to force the level set to segment only the linear shapes. This is done by adding a term $E_{\text {Line }}$ that measures how well the level set represents the line. The new energy functional becomes:

$$
E=E_{\text {Seg }}+\alpha E_{\text {Line }} .
$$

To derive $E_{\text {Line }}$ the line is represented by its polar coordinates:

$$
\rho=x \cos \theta+y \sin \theta,
$$

where $\theta$ is the orientation of the normal to the line with the $x$ axis, and $\rho$ is the distance of the line from the origin. The square distance, $r^{2}$, of a point $\left(x_{1}, y_{1}\right)$ from the line is obtained by plugging the coordinates of the point into (9):

$$
r^{2}=\left(x_{1} \cos \theta+y_{1} \sin \theta-\rho\right)^{2} .
$$

So we can express $E_{\text {Line }}$ which minimizes the sum of distances between the points inside the zero level set and a line with parameters $(\rho, \theta)$ : 

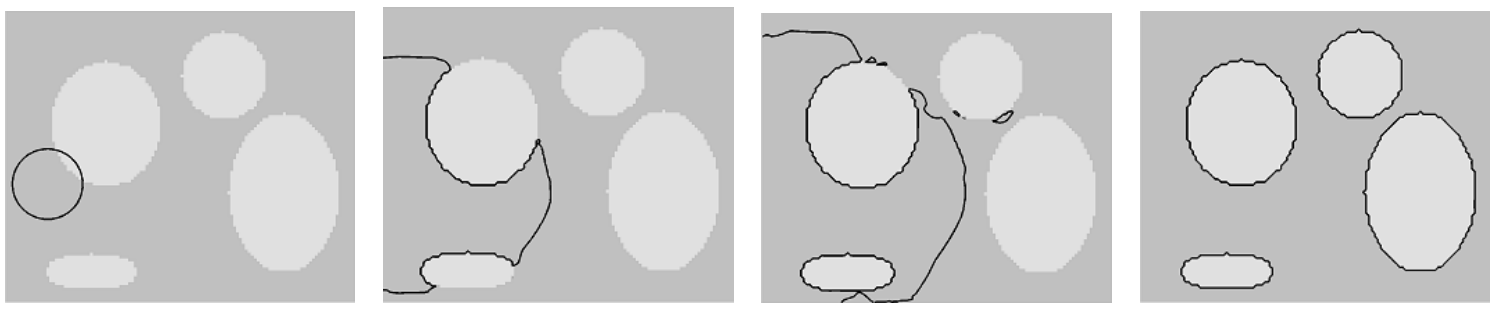

Figure 1. Evolution of the boundary for the Chan-Vese level set (with a single level set function). Due to the implicit level set representation, the topology is not constrained, which allows for splitting and merging of the boundary.

$$
E_{\text {Line }}(\rho, \theta, \phi)=\int_{\Omega}(\rho-x \cos \theta-y \sin \theta)^{2} H_{\varepsilon}(\phi) d x d y .
$$

If the points inside the zero level set represent a line, $E_{\text {Line }}$ will tend to be zero.

Keeping $\rho$ and $\theta$ constant and minimizing this energy functional (11) with respect to $\phi$, we deduce the associated Euler-Lagrange equation for $\phi$ as

$$
\frac{\partial E_{\text {Line }}}{\partial \phi}=\delta_{\varepsilon}(\phi)\left[(\rho-x \cos \theta-y \sin \theta)^{2}\right] .
$$

Keeping $\phi$ fixed and setting $\frac{\partial E_{\text {Line }}}{\partial \rho}=0$, and $\frac{\partial E_{\text {Line }}}{\partial \theta}=0$, it is straightforward to solve for the line's $\rho$ and $\theta$ parameters as:

$$
\rho=\bar{x} \cos \theta+\bar{y} \sin \theta,
$$

where $\bar{x}$ and $\bar{y}$ represent the centroid of the region inside the zero level set and given by [26, 27]:

$$
\bar{x}=\frac{\int_{\Omega} x H_{\varepsilon}(\phi) d x d y}{\int_{\Omega} H_{\varepsilon}(\phi) d x d y}, \bar{y}=\frac{\int_{\Omega} y H_{\varepsilon}(\phi) d x d y}{\int_{\Omega} H_{\varepsilon}(\phi) d x d y},
$$

and

$$
\theta=\frac{1}{2} \arctan \left(\frac{a_{2}}{a_{1}-a_{3}}\right)
$$

where $a_{1}, a_{2}$ and $a_{3}$ are given by [26]

$$
\begin{aligned}
& a_{1}=\int_{\Omega}(x-\bar{x})^{2} H_{\varepsilon}(\phi) d x d y, \\
& a_{2}=2 \int_{\Omega}(x-\bar{x})(y-\bar{y}) H_{\varepsilon}(\phi) d x d y, \\
& a_{3}=\int_{\Omega}(y-\bar{y})^{2} H_{\varepsilon}(\phi) d x d y .
\end{aligned}
$$

The Euler-Lagrange equation for the total functional (8) can now be implemented by the following gradient descent:

$$
\frac{\partial \phi}{\partial t}=\delta_{\varepsilon}(\phi)\left[\mu \operatorname{div}\left(\left(\frac{\nabla \phi}{|\nabla \phi|}\right)\right)-v-\lambda_{1}\left(f-c_{1}\right)^{2}+\lambda_{2}\left(f-c_{2}\right)^{2}-\alpha(\rho-x \cos \theta-y \sin \theta)^{2}\right],
$$

where the scalars $c_{1}, c_{2}, \rho$, and $\theta$ are updated with the level set evolution according to Eqs. $(6,7,13-18)$. 
The weights $\lambda_{1}$ and $\lambda_{2}$ can be used to speed up the evolution towards the object boundaries, while $\mu$ and $v$ regulate the zero level set. For example $\mu$ has a scaling role [1]; if we have to detect all or as many objects as possible and of any size, then $\mu$ should be small. If we have to detect only larger objects, and not to detect smaller objects (like points, due to the noise), then $\mu$ has to be larger. The weight $\alpha$ controls the emphasize on the required object shape.

It has been observed in our experiments that for sufficiently large $\alpha$, the final shape of the zero level set for a nonlinear object will be the axis of second moment (axis of elongation) for the object as illustrated in Fig.2. To get around this, the weight of the area term, $v$, in (2), and consequently (8), is increased. This causes the minimum of the energy functional (8) to occur when $\phi<0$ all over the image, thus ignoring the undesired object.

\section{Multi-Object Segmentation}

The previous method works only if there is one object in the image. If this object is linear, it will be detected, whereas other shapes are ignored. If there are more than one object, $H(\phi)$ will represent all those objects and Equations (13-18) will not be applicable. In this section we extend our method in order to perform multiple region segmentation based on fuzzy memberships that are computed by a Fuzzy C-mean algorithm (FCM) [19].

\subsection{The Fuzzy C-mean Algorithm}

The (FCM) generalizes the hard k-means algorithm to allow a point to partially belong to multiple clusters. Therefore, it produces a soft partition for a given dataset. If we assumed that $U[c \times n]$ is a membership matrix which contains the degree of membership for each cluster. Here, $n$ denotes the number of patterns and $c$ the number of clusters. In general the elements $u_{i k}$ of the matrix $U$ are in the interval $[0,1]$ and denote the degree of membership of the pattern $\mathbf{x}_{k}$ to the cluster $c_{i}$. The following condition must be satisfied

$$
\sum_{i=1}^{c} u_{i k}=1, \forall 1 \leq k \leq n
$$

Also if we assumed that $\mathbf{V}=\left(\mathbf{v}_{1}, \mathbf{v}_{2}, \ldots, \mathbf{v}_{c}\right)$ is a vector of cluster centre to be identified. The (FCM) attempts to cluster feature vectors by searching for local minima of the following objective function [19]:

$$
J_{m}(U, \mathbf{v})=\sum_{i=1}^{c} \sum_{k=1}^{n}\left(u_{i k}\right)^{m} D_{i k},
$$

where the real number $m \in[0, \infty)$ is a weighting exponent on each fuzzy membership (typically taken equal to 2), $D_{i k}$ is some measure of similarity between $v_{i}$ and $\mathbf{x}_{k}$ or the attribute vectors and the cluster centers of each region. Minimization of $J_{m}$ is based on the suitable selection of $U$ and $\mathbf{V}$ using an iterative process through the following equations:

$$
\begin{aligned}
& U_{i k}=\left(\sum_{j=1}^{c}\left(\frac{D_{i k}}{D_{j k}}\right)^{\frac{2}{(m-1)}}\right)^{-1} \forall i, k, \\
& \mathbf{v}_{i}=\frac{\sum_{k=1}^{n} u_{i k}{ }^{m} \mathbf{x}_{k}}{\sum_{k=1}^{n} u_{i k}{ }^{m}} \forall i .
\end{aligned}
$$

The algorithm stops when $u_{i k(\alpha)}-u_{i k(\alpha-1)}<\varepsilon$, or the maximum number of iteration has been reached. The (FCM) has several advantages. 1) It is unsupervised, 2) it can be used with any number of features and any 

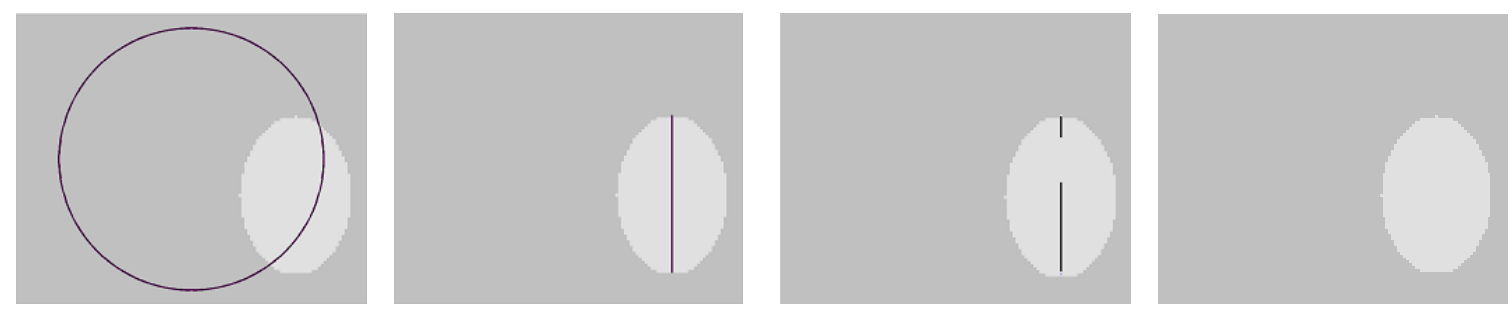

Figure 2. Evolution of the boundary for the level set under the functional (8). Due to the $E_{\text {Line }}$ term, the final shape of the boundary is the second moment axis of the object. Increasing $v$ causes smaller part of the object axis be detected. Further increase in $v$ leaves the nonlinear object undetected.

number of classes and 3) it distributes the membership values in a normalized fashion. However, being unsupervised, it is not possible to predict ahead of time what type of clusters will emerge from the FCM.

\subsection{Handling Multiple Objects}

The FCM algorithm provides an initial segmentation of the image into a given number $N$ of clusters. Let $u_{i}(x, y)$ denotes the membership of the pixel $(x, y)$ in the i-th cluster. A level-set function, $\phi_{i}$, is associated with each cluster, except for the cluster with largest number of pixels as it is assumed to be the background. Each $\phi_{i}$ is initialized such that:

$$
\left\{\begin{array}{l}
\phi_{i}>0, \forall(x, y) \in\left\{u_{i}(x, y) \geq 0.5\right\} \\
\phi_{i}<0, \text { Otherwise }
\end{array}\right.
$$

The term $E_{\text {Line }}$ of the energy functional (8) is still given by (11), whereas the term $E_{\text {seg }}$ is now based on minimizing several level set functions $\left\{\phi_{i}\right\}$ :

$$
E_{\text {seg }}(\phi)=\sum_{i=1}^{N-1}\left[\lambda \int_{\Omega}\left[\left(1-u_{i}\right) H_{\varepsilon}\left(\phi_{i}\right)+u_{i}\left(1-H_{\varepsilon}\left(\phi_{i}\right)\right)\right] d x+\mu \int_{\Omega}\left|\nabla H_{\varepsilon}\left(\phi_{i}\right)\right| d x+v \int_{\Omega} H_{\varepsilon}\left(\phi_{i}\right) d x\right],
$$

which can be simplified to

$$
E_{\text {seg }}(\phi)=\sum_{i=1}^{N-1}\left[\lambda \int_{\Omega}\left(1-2 u_{i}\right) H_{\varepsilon}\left(\phi_{i}\right) d x+\mu \int_{\Omega}\left|\nabla H_{\varepsilon}\left(\phi_{i}\right)\right| d x+v \int_{\Omega} H_{\varepsilon}\left(\phi_{i}\right) d x\right],
$$

where $\mu, \lambda$ are regularizing parameters to be estimated or chosen a priori. The functional (26) aims to maximize the total membership inside the isocontour of the zero level-set.

The Euler-Lagrange PDE for the new energy functional can now be implemented by the following gradient descent for each level set:

$$
\frac{\partial \phi_{i}}{\partial t}=\delta_{\varepsilon}\left(\phi_{i}\right)\left[\mu \operatorname{div}\left(\left(\frac{\nabla \phi_{i}}{\left|\nabla \phi_{i}\right|}\right)\right)-v-\lambda(1-2 u)-\alpha\left(\rho_{i}-x \cos \theta_{i}-y \sin \theta_{i}\right)^{2}\right],
$$

where the scalars $\rho_{i}$, and $\theta_{i}$ are updated with the level set evolution according to (13)-(18). The overall level set representation is eventually obtained from the final $\left\{\phi_{i}\right\}$ as $\max \left(\phi_{i}\right)$, for all $\mathrm{i}$.

One problem however may arise if multiple disjoint objects belong to the same cluster (e.g., if they have the same color). Therefore after the initial clustering by the FCM algorithm, connected-component labeling is carried out on a hardened version of the result so objects within the same cluster are separated. Each object part is represented by an image that contains the membership information but with other twin object replaced by 0 . Note that $N$ in (26) will thus be increased accordingly.

We summarize the above scheme by the following algorithm outline: 
- The input image is initially segmented into a number of clusters based on the FCM algorithm.

- Connected-component labelling is carried out on hardened version of each cluster, except the background, to separate the objects in that cluster.

- Construct an image for each initially detected object, this image contains the membership information of that object.

- Initial level-set is imposed for each object according to (24).

- Each level set is evolved based on (27). If the object is not linear, the isocontour of the zero level-set associated with this object will vanish.

- At the end of evolution, the final level set equals $\phi=\max \left\{\phi_{i}\right\}$, for all i. The remaining level sets, $\left\{\phi_{i}\right\}$, represent the linear objects. The parameters of the linear objects have been already calculated during the evolution according to (13)-(18).

This algorithm presents a simple, yet effective method to handle multiple objects in images. This is in contrast to existing methods that mostly did not consider this case. One of the few reported techniques that did consider it is the one by Brox et al. [25]. However, their method is rather complicated and not straightforward to implement because they employed the combination of several ideas from multi-scale basis, a divide-and-conquer strategy, expectation-maximization principle and nonparametric Parzen density estimation.

\section{Experimental Results}

In order to evaluate the performance of the proposed technique on line segmentation, several experiments using synthetic and real images have been carried out. In the experiments, we choose the regularizing parameters as follows: $\alpha=1, \lambda=10, \mu=0.5$, and $v=10$. As our method is region-based segmentation it is robust in noisy images. This is demonstrated in Fig. 3. All lines have been successfully extracted from an image artificially corrupted with high noise with standard deviation $\sigma=45$. Note that due to the shape constraints, our method again extracts only the lines and ignores other objects. For the sake of comparison, the result of the classical Hough transform applied to the same test image without noise, is shown in Fig. 3(c). Apparently, the level set method extracts only linear objects in the image, whereas Hough transform can also detect linear boundaries of objects (e.g., the box). However once the noise level in the image increases, Hough transform will face some problems. This is, because it depends largely on edge detection, it is sensitive to image noise, which may result in missing legitimate image lines when applied to the image in Fig. 3(a), as shown in Fig. 3(d).

We use the proposed method to extract intersected lines that have different colors; as shown in Fig. 4, which is a difficult problem for almost all existing level-set-based methods. Because of the lines intersections, the three lines could be treated as one object that would not become linear anymore. The initial level-sets based on the FCM output according to (24) are imposed on the image in Fig. 4(b). As shown in Fig. 4(c), the proposed method successfully segmented the lines, in spite of their intersections, and because of the shape-based term, other objects were discarded.

An example of a real image is illustrated in Fig. 5. The FCM output, shown in Fig. 5(b), treats lines and birds as the same object. This is clear in Fig. 5(c) where the initial level sets take the lines and birds as one object. The level sets correct this and successfully extract only the lines in Fig. 5(d), even in spite of the birds touching the lines. Another real example is considered in Fig. 6. The initial level set based on the FCM algorithm is shown in Fig. 6(b). The final result in Fig. 6(c) shows how our algorithm can extract only the linear objects and discard the others. 


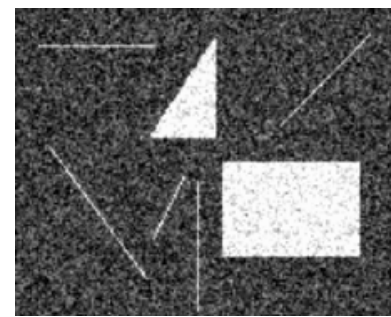

(a)

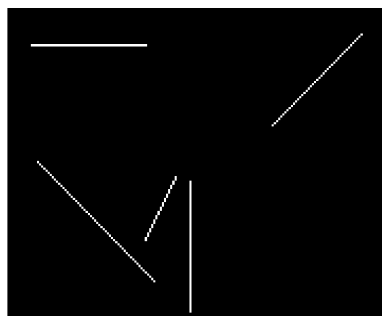

(b)

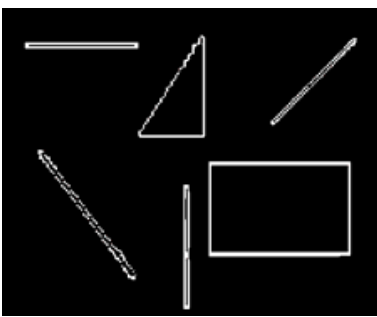

(c)

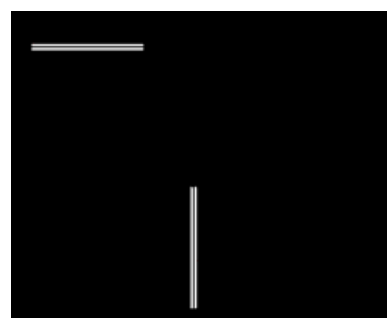

(d)

Figure 3. Extracted lines from highly noisy image (noise standard deviation $=45$ ), (a) Input image, (b) The final result using our method, (c) Result of Hough transform on the noise-free image, (d) Result of Hough transform on the noisy image.

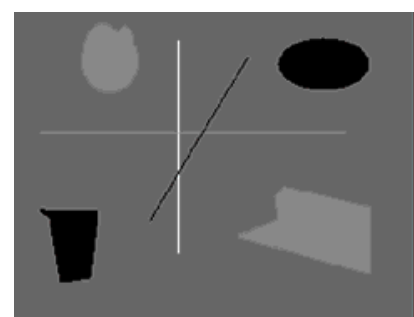

(a)

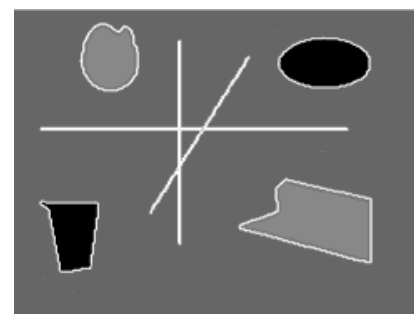

(b)

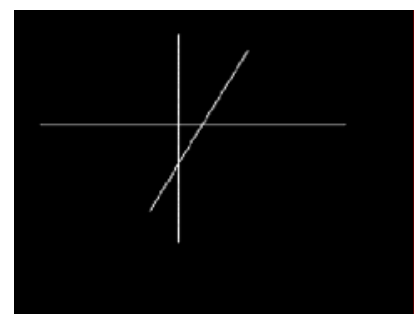

(c)

Figure 4. Extraction of intersected lines with different intensities. (a) The input image, (b) Initial level set based on FCM clustering , (c) Final result.

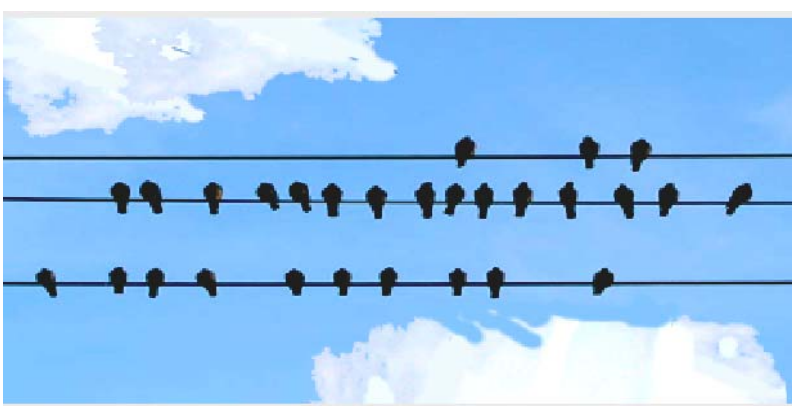

(a)

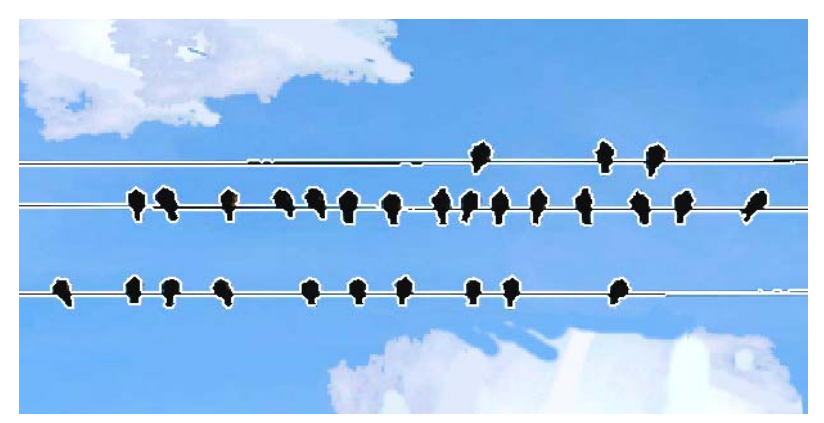

(c)

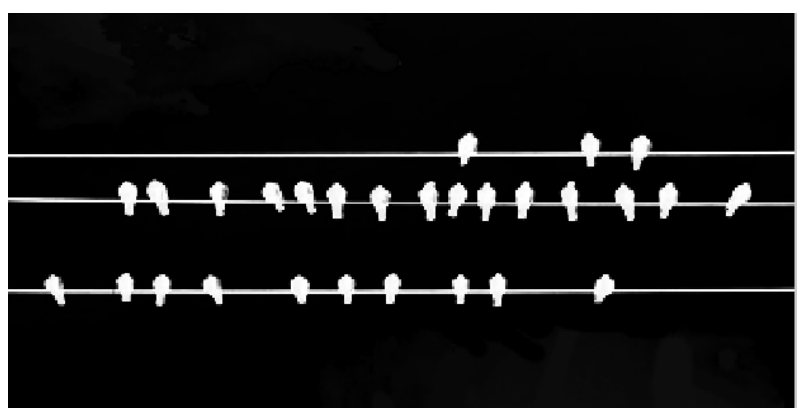

(b)

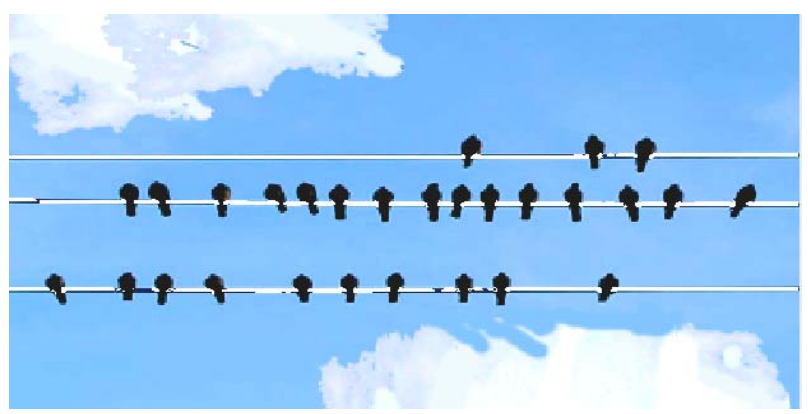

(d)

Figure 5. A real image "Birds on power lines". (a) Input image, (b) Hardened output of FCM algorithm, (c) Initial level set based on the output of FCM algorithm, (d) Final level set showed how our method excluded the birds. 


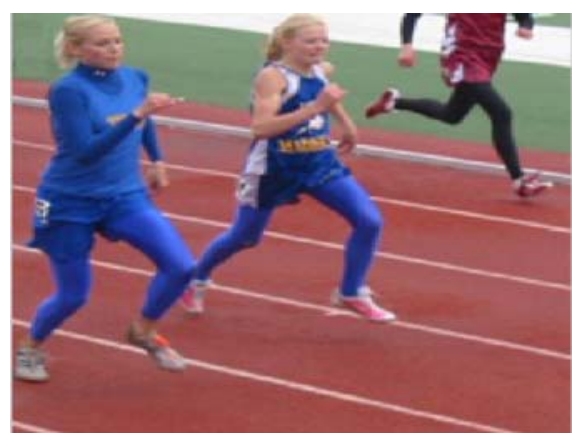

(a)

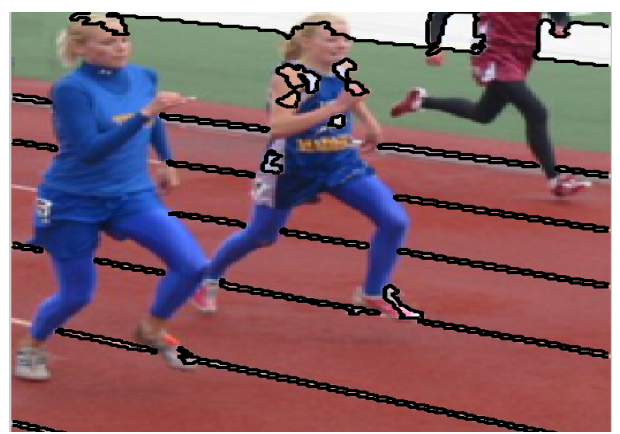

(b)

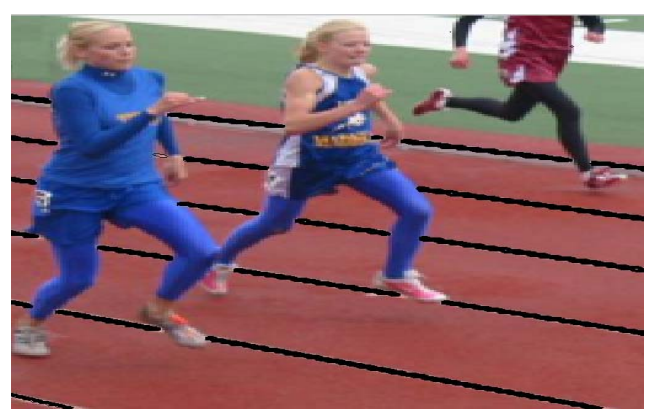

(c)

Figure 6. A real image "A running track". (a) Input image, (b) Initial level set based on the output of FCM algorithm, (c) The final level set imposed on the image.

\section{Application: Lens Distortion Removal}

In this section we apply the variational-based method in the previous sections to calibrate lens distortion. We will focus in this section on recovering the radial component of lens distortion, as it is often the most prominent in images. Our approach is based on the analysis of distorted images of straight lines. We use a PDE-based level set method to find the lens distortion parameters that straighten these lines. One key advantage of this method is that it integrates the extraction of image distorted lines and the computation of distortion parameters within one energy functional which is minimized during level set evolution. Thus our approach, unlike most other nonmetric calibration methods [21, 22, 23], avoids the propagation of errors in feature extraction onto the computation stage. This results in a more robust computation even at high noise levels.

The closest work to ours is that of Kang [24]. He used the traditional snake to calculate the radial lens distortion parameters. However, his method is sensitive to the location of the initial contour, so the user should specify the position of the initial contour. In contrast, our level-set based method has some global convergence property that makes it not sensitive to the initial level set.

We start by giving briefly a standard model for lens distortion in camera lenses, and then we formulate our approach.

\subsection{Camera Distortion Model}

The standard model for the radial and decentering distortion [20,21, 28] is mapping from the observable, distorted image coordinates, $(x, y)$, to the unobservable, undistorted image plan coordinates, $\left(x^{u}, y^{u}\right)$. Neglecting all coefficients other than the first radial distortion term, the model becomes:

$$
\begin{aligned}
& x^{u}=x+\hat{x}\left(\kappa r^{2}\right), \\
& y^{u}=y+\hat{y}\left(\kappa r^{2}\right),
\end{aligned}
$$


$\widehat{x}=x-c_{x}, \hat{y}=y-c_{y}, r^{2}=\widehat{x}^{2}+\hat{y}^{2}$, and $\kappa$ is the coefficient of radial distortion. $r$ is the radius of an image point from the distortion center, defined as $\left(c_{x}, c_{y}\right)$ above. The distortion centre is quite often located near the image centre $[20,22,28]$. Following pervious works $[24,28]$ we assume the distortion centre to be the image centre. We thus seek to recover, $\kappa$, as it has the most dominating effect. So the distortion model becomes:

\subsection{Our Approach}

Our goal here is to use the energy functional (8) in order to force the level set to segment linear, or should-to-be-linear, objects from the image and simultaneously solve for the lens distortion parameter. The algorithm outlined in Section 4 is used here. However the $E_{\text {Line }}$ term of the energy functional becomes

$$
E_{\text {Line }}\left(\rho_{i}, \theta_{i}, \phi_{i}\right)=\int_{\Omega}\left(\rho_{i}-x^{u} \cos \theta_{i}-y^{u} \sin \theta_{i}\right)^{2} H\left(\phi_{i}\right) d x d y,
$$

which measures how well a level set presents a line in the undistorted image coordinates $\left(x^{u}, y^{u}\right)$, with $\theta_{i}$ being the orientation of the normal to the line, and $\rho_{i}$ being the distance to the line from the origin. Note that the undistorted coordinates are related to the given distorted image coordinates $(x, y)$ via the distortion parameter $\kappa$ as in (28). As for $\kappa$ that minimizes the total energy functional $E$, we start with an initial guess $\kappa^{0}$ (in our implementation, we take it 0 ). Introducing an artificial time, $t, \kappa$ is then updated according to the gradient decent rule $\frac{\partial \kappa}{\partial t}=-\frac{\partial E}{\partial \kappa}$, where

$$
\frac{\partial E}{\partial \kappa}=2 \alpha \sum_{i=1}^{N-1} \int_{\Omega}\left(x^{u} \cos \theta_{i}+y^{u} \sin \theta_{i}-\rho_{i}\right)\left[\left(x-c_{x}\right) r^{2} \cos \theta_{i}+\left(y-c_{y}\right) r^{2} \sin \theta_{i}\right] H\left(\phi_{i}\right) d x d y .
$$

Note that $\kappa$ is updated based on all level sets, but on the other hand each level set is updated by deducing the associated Euler-Lagrange equation for $\phi_{i}$ :

$$
\left.\frac{\partial \phi_{i}}{\partial t}=-\frac{\partial E}{\partial \phi_{i}}=\delta_{\varepsilon}\left(\phi_{i}\right)\left[\mu \operatorname{div}\left(\frac{\nabla \phi_{i}}{\left|\nabla \phi_{i}\right|}\right)\right)-v-\lambda\left(1-2 u_{i}\right)-\alpha\left(\rho_{i}-x^{u} \cos \theta_{i}-y^{u} \sin \theta_{i}\right)^{2}\right],
$$

where the scalars $\rho_{i}, \theta_{i}$, and $\kappa$ are updated with the level set evolution according to $(13,15,31)$. In the steady state the value of $\kappa$ is the required lens distortion coefficient.

\subsection{Experimental Results}

The approach is applied to real images acquired by a cheap BenQ camera. To calibrate the radial lens distortion coefficient, we captured an image of a group of straight lines on a white paper; see Fig. 7(a). Such a calibration pattern is easily prepared (e.g., with just a printer) without any special construction overhead. Another sample image captured by the same camera is shown in Fig. 7(b). Both acquired images are $160 \times 120$ and have noticeable lens distortion. Our approach is then applied to the calibration image to recover the value of lens distortion parameter. Figs. 7(c-d) show the initial and final zero-level sets, respectively. Our method took less than a minute on $\mathrm{P} 42.8 \mathrm{GHz}$ pc. The estimated $\kappa$ is employed to remove the distortion from the original images taken by the camera, see Fig. 7(e-f). Clearly the should-to-be image lines are indeed mapped to straight lines in the resultant images. One may notice some artifacts (left intentionally) with the undistorted images due to the inverse mapping of the distortion model in (28), which can be fairly fixed, if desired, by doing some post-processing. Further experiments on synthetic data, [29], have shown that the accuracy of our proposed method remains within 0.1 pixels up to a high noise level of $\sigma \cong 35$. 


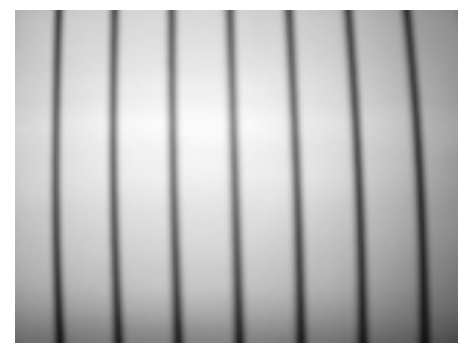

(a)

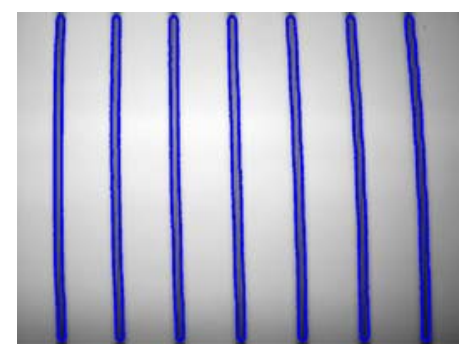

(d)

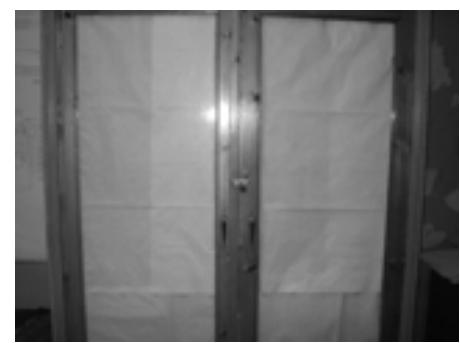

(b)

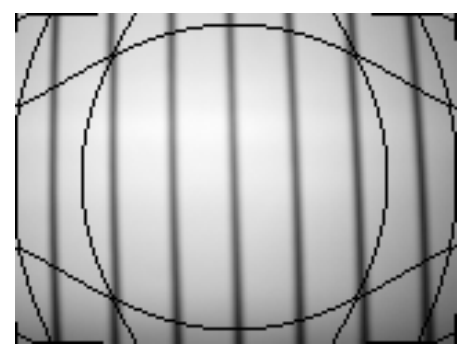

(e)

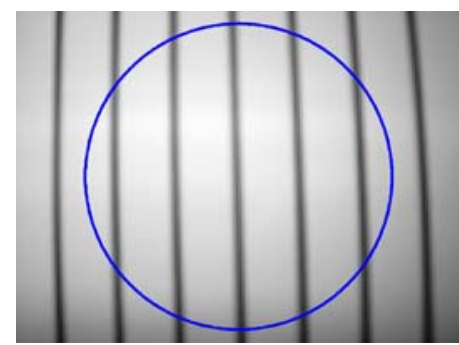

(c)

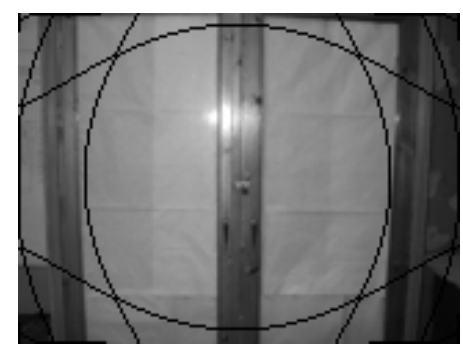

(f)

Figure 7. Lens distortion removal from a real images: (a) The calibration image which is used to get $\kappa$, (b) An input distorted image, (c) Initial zero level set, (d) Final zero level set (e) Calibration image undistorted, (f) Image in (b) undistorted using the obtained $\kappa$.

\section{Conclusions}

We have presented a new variational approach to integrate parametric shapes into level set-based segmentation. In particular, we addressed the problem of extracting linear image objects, selectively, while other image objects are ignored. Our method is inspired by ideas introduced by Chan and Vese by formulating a new energy functional taking into account the line parameters. By simultaneously minimizing the proposed energy functional with respect to the level set function and the line parameters, the linear shapes are detected while the line parameters are obtained. This method is extended using Fuzzy memberships to segment simultaneous lines of different intensities. This method is shown experimentally to segment simultaneous lines of different intensities, even in images of large noise.

We have also applied the proposed approach to calibrate camera lens distortion. In order to achieve this, the formulated energy functional depends on the parameters of lens distortion parameters as well. By evolving the level functions minimizing that energy functional, the image lines and lens distortion parameters are obtained. All this approach needs is an image captured by the camera for a group of straight lines on a white paper. Such a calibration pattern is easily prepared (e.g., with just a printer) without any special construction overhead. One key advantage of our method is that it integrates the extraction of image features needed for calibration and the computation of distortion parameters; thus avoiding, unlike most other nonmetric calibration methods, the propagation of errors in feature extraction onto the computation stage.

Our future research is directed towards the segmentation of other parametric shapes from images, e.g., conics, which are of special importance in geometric computer vision. In addition, it is directed to incorporating more lens distortion parameters in order to be able to remove the distortion from severelydistorted images, such as in very-wide-view cameras, and to achieve more accurate calibration. 


\section{References}

[1] T. Chan and L. Vese, “Active contours without edges”, IEEE Trans. Image Processing, 2001, pp. 266-277.

[2] L. Staib and J. Duncan. "Boundary finding with parametrically deformable models”, IEEE Trans. on Patt. Anal. and Mach. Intel., 1992, pp. 1061-1075, 1992.

[3] T. Cootes, A. Hill, C. Taylor, and J. Haslam, "Use of active shape models for locating structures in medical images”, Image and Vision Computing, 1994, pp. 355-365.

[4] M. Leventon, W. L. Grimson, and O. Faugeras, "Statistical shape influence in geodesic active contours”, in Proc. Conf. Computer Vis. and Pattern Recog., volume 1, Hilton Head Island, SC, June 13-15, 2000, pp. 316-323.

[5] A. Tsai, A. Yezzi, W. Wells, C. Tempany, D. Tucker, A. Fan, E. Grimson, and A.. Willsky, "Model-based curve evolution technique for image segmentation", in Conf. on Comp. Vision and Patt. Recog., Kauai, Hawaii, 2001, pp. 463-468.

[6] D. Cremers, F. Tischhäuser, J. Weickert, and C. Schnörr, "Diffusion snakes: introducing statistical shape knowledge into the Mumford-Shah functional”, Int. J. of Comp. Vision, 2002, pp.295-313.

[7] D. Cremers, T. Kohlberger, and C. Schnörr, "Nonlinear shape statistics in Mumford-Shah based segmentation", in A. Heyden et al., editors, Proc. of the Europ. Conf. on Comp. Vis., Copenhagen, May 2002, volume 2351 of LNCS, pp. 93-108,

[8] M. Rousson and N. Paragios, "Shape priors for level set representations", in A. Heyden et al., editors, Proc. of the Europ. Conf. on Comp. Vis., Copenhagen, May 2002, volume 2351 of LNCS, pp. 78-92.

[9] S. Osher and J. Sethian, "Fronts propagation with curvature dependent speed: Algorithms based on Hamilton-Jacobi formulations", J. of Comp. Phys., 1988, pp. 12-49.

[10] V. Caselles, R. Kimmel, and G. Sapiro, "Geodesic active contours", in Proc. IEEE Internat. Conf. on Comp. Vision, Boston, USA, 1995, pp. 694-699.

[11] S. Kichenassamy, A. Kumar, P. Olver, A. Tannenbaum, and A. Yezzi, "Gradient flows and geometric active contour models", in Proc. IEEE International Conf. on Comp. Vision, Boston, USA, 1995, pp. 810-815.

[12] Y. Chen, S. Thiruvenkadam, H. Tagare, F. Huang, D. Wilson, and E. Geiser, "On the incorporation of shape priors into geometric active contours", in IEEE Workshop on Variational and Level Set Methods, Vancouver, CA, 2001, pp. 145-152.

[13] D. Cremers, N. Sochen and C. Schnörr, "Towards recognition-based variational segmentation using shape priors and dynamic labeling" in 4th Int. Conf. on Scale Space Theories in Computer Vision, Isle of Skye, June 2003, LNCS Vol. 2695, pp. 388-400.

[14] X. Pardo , V. Leboran and R. Dosil, “Integrating prior shape models into level-set approaches”, in Pattern Recognition Letters, Elsevier Science, 2004, pp. 631-639.

[15] S. Osher and R. Fedkiw, Level set methods and dynamic implicit surfaces, Springer-Verlag, USA, 2003.

[16] S. Osher and N. Paragios, Geometric level set methods in imaging, vision and graphics, Springer Verlag, USA, 2003.

[17] J. Sethian, Level set methods and fast marching methods: evolving interfaces in computational geometry, fluid mechanisms, computer vision, and material science, Cambridge University Press, 1999.

[18] O. Faugeras, Three dimensional computer vision: a geometric viewpoint, Cambridge, MA: MIT Press, 1993.

[19] J. C. Bezdek and P. F. Castelaz, "Prototype Classification and Feature Selection with Fuzzy Sets”, IEEE Trans. On Systems, Man and Cybernetics vol. SMC-7, pp 87-92, 1977. 
[20] J.Weng, P. Cohen, and M. Herniou, "Camera calibration with distortion models and accuracy evaluation”, PAMI, 14(10), Oct 1992.

[21] F. Devernay and O. Faugeras, "Straight lines have to be straight: automatic calibration and removal of distortion from scenes of structured environments", Machine Vision and Applications, Vol. 1, 14-24, 2001.

[22] B. Prescott and G. McLean, "Line-based correction of radial lens distortion", Graphical Models and Image Processing, 59(1):39-47, 1997

[23] R. Swaminathan and S. Nayar, "Non-metric calibration of wide-angle lenses and polycameras", IEEE Trans. Pattern Analysis and Machine Intelligence (PAMI), 22(10), Oct. 2000.

[24] S. Kang, "Radial distortion snakes", IAPR Workshop on Machine Vision Applications (MVA2000), Tokyo, Japan, Nov. 2000, pp. 603-606.

[25] T. Brox and J. Weickert, "Level Set Based Image Segmentation with Multiple Regions", In Pattern Recognition, Springer LNCS 3175, pp. 415-423, Tübingen, Germany, Aug. 2004.

[26] R. Jain, R. Kasturi and B. Schunck, Machine vision, McGraw-Hill, USA, 1995.

[27] D. Cremers, S. Osher, S. Soatto, "Kernel density estimation and intrinsic alignment for shape priors in level set segmentation”, International Journal of Computer Vision, 69(3), pp. 335-351, 2006.

[28] M. Ahmed and A. Farag, "Nonmetric calibration of camera lens distortion: differential methods and robust estimation", IEEE Trans. on image processing, vol. 14, no. 8, pp. 1215-1230, 2005.

[29] M. El-Melegy and N. Al-Ashwal, "Lens distortion calibration using level sets", Lecture Notes in Computer Science, N. Paragios et al. (Eds.)., Springer-Verlag, Berlin, LNCS 3752, pp. 356 - 367, 2005. 